\title{
Solutions to the tethered galaxy problem in an expanding universe and the observation of receding blueshifted objects
}

\author{
Tamara M. Davis $⿴$ Charles H. Lineweaver \\ Department of Astrophysics, University of New South Wales, Sydney, 2052, Australia
}

\begin{abstract}
We use the dynamics of a galaxy, set up initially at a constant proper distance from an observer, to derive and illustrate two counter-intuitive general relativistic results. Although the galaxy does gradually join the expansion of the universe (Hubble flow), it does not necessarily recede from us. In particular, in the currently favored cosmological model, which includes a cosmological constant, the galaxy recedes from the observer as it joins the Hubble flow, but in the previously favored cold dark matter model, the galaxy approaches, passes through the observer, and joins the Hubble flow on the opposite side of the sky. We show that this behavior is consistent with the general relativistic idea that space is expanding and is determined by the acceleration of the expansion of the universe - not a force or drag associated with the expansion itself. We also show that objects at a constant proper distance will have a nonzero redshift; receding galaxies can be blueshifted and approaching galaxies can be redshifted.
\end{abstract}

\section{INTRODUCTION}

The interpretation of the expansion of the universe in general relativistic cosmology was, and to some extent still is, the subject of discussion and controversy. Robertson ${ }^{1}$ and Walker $^{2}$ presented the metric for a homogeneous expanding isotropic universe with a comoving frame in which receding bodies are at rest, and peculiar velocities are velocities measured with respect to this comoving frame. This standard metric and the picture of expanding and curved space is fully consistent with special relativity locally and general relativity globally. ${ }^{3}$ Milne rejected the expansion of space and insisted instead on expansion through space and introduced Newtonian cosmology 4 Although the original formulation was found to be logically inconsistent,,$\frac{5}{-}$ many different formulations of Newtonian cosmology have since been proposed 6 Recession velocities are a fundamental feature of the general relativistic expansion of the universe. Harrison ${ }^{3}$ has pointed out a conflict in the use of recession velocities that is resolved when a distinction is made between the empirical and theoretical Hubble laws: the empirical redshift distance relation, $c z=H D$, is valid only at low redshifts, while $v=H D$ derived from the RobertsonWalker metric is valid for all distances. ( $H$ is Hubble's constant, $v$ is the recession velocity, $z$ is the redshift, $c$ is the speed of light, and $D$ is the proper distance.) Perhaps partly because it appears paradoxical and partly because of the different definitions of distance, recession velocities greater than $c$ are still a source of much confusion and skepticism, ${ }^{7}$ despite several attempts to clarify the issue. 8

Recently it has been argued that the expansion of space is a peculiarity of the particular coordinate system used, and the expansion can equally well be described as an expansion through space,$\frac{9}{9}$ or alternately, that the expansion is locally kinematical ${ }^{10}$ Debate persists over what spatial scales participate in the expansion of the universe, 11.12 and the effect of the expansion of the universe on local systems is a topic of current research. 13
The general expansion of the universe is known as the Hubble flow. A persistent confusion is that galaxies set up at rest with respect to us and then released will start to recede as they pick up the Hubble flow. This confusion mirrors the assumption that, without a force to hold them together, galaxies (and our bodies) would be stretched as the universe expands. The aim of this paper is to clarify the nature of the expansion of the universe, including recession velocities and cosmological redshifts, by looking at the effect of the expansion on objects that are not receding with the Hubble flow. This paper is an extension of previous discussions on the expansion of space. 11,14

To clarify the influence of the expansion of the universe, we consider the "tethered galaxy" problem. 15,16 We set up a distant galaxy at a constant distance from us and then allow it to move freely. The essence of the question is, once it has been removed from the Hubble flow and then let go, what effect, if any, does the expansion of the Universe have on its movement? In Sec. II we derive and illustrate solutions to the tethered galaxy problem for arbitrary values of the density of the universe $\Omega_{\mathrm{M}}$ and the cosmological constant $\Omega_{\Lambda}$. We show that no drag is associated with unaccelerated expansion. Our calculations agree with and generalize the results obtained by Peacock, 16 but we also point out an interesting interpretational difference.

The cosmological redshift is important because it is the most readily observable evidence of the expansion of the universe. In Sec. III we point out a consequence of the fact that the cosmological redshift is not a special relativistic Doppler shift, and we derive the counter-intuitive result that a galaxy at a constant proper distance will have a nonzero redshift. In Sec. IV] we summarize our results and discuss relativistic radio jets as examples of receding blueshifted objects. 

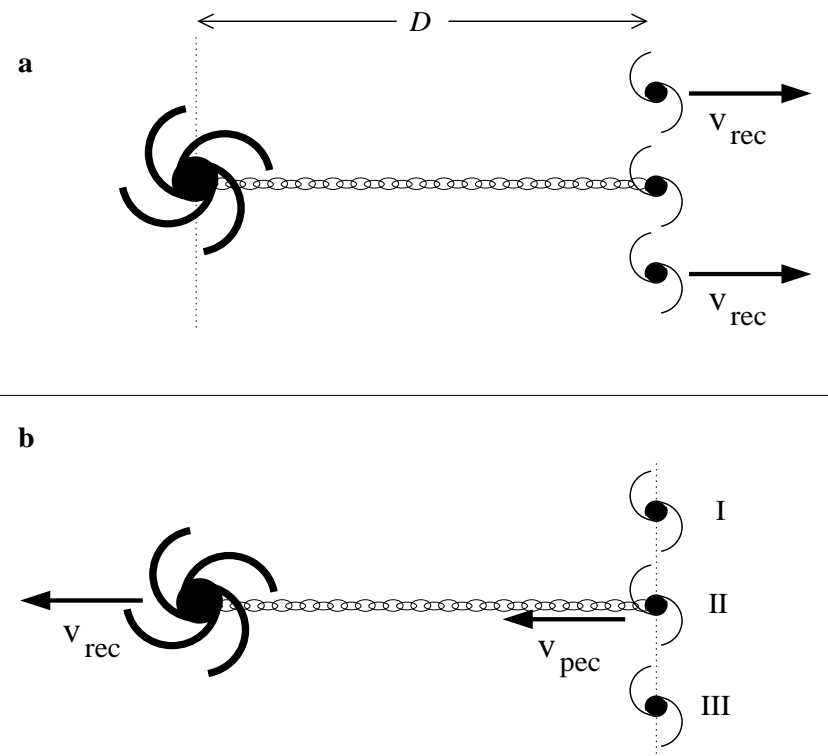

FIG. 1: (a) A small distant galaxy (considered to be a massless test particle) is tethered to an observer in a large galaxy. The proper distance to the small galaxy, $D$, remains fixed; the small galaxy does not share the recession velocity of the other galaxies at the same distance. The tethered galaxy problem is "What path does the small galaxy follow when we unhook the tether?" (b) Drawn from the perspective of the local comoving frame (out of which the test galaxy was boosted), the test galaxy has a peculiar velocity equal to the recession velocity of the large galaxy. Thus, the tethered galaxy problem can be reduced to "How far does an object, with an initial peculiar velocity, travel in an expanding universe?"

\section{THE TETHERED GALAXY PROBLEM}

We assume a homogeneous, isotropic universe and use the standard Friedman-Robertson-Walker (FRW) metric ${ }^{10}$ We only encounter radial distances, and therefore the FRW metric can be simplified to

$$
d s^{2}=-c^{2} d t^{2}+a^{2}(t) d \chi^{2},
$$

where $t$ is the proper time of each fundamental observer (also known as the cosmic time) $\stackrel{17}{!}$ The scale factor of the universe, $a$, is normalized to 1 at the present day, $a\left(t_{0}\right)=$ $a_{0}=1$, and $\chi$ is the comoving coordinate. The proper distance, $D=a \chi$, is the distance (along a constant time surface, $d t=0$ ) between us and a galaxy with comoving coordinate $\chi$. This is the distance a series of comoving observers would measure if they each laid their rulers end to end at the same cosmic instant 17 Differentiation with respect to proper time is denoted by a dot and is used to define "approach" $(\dot{D}<0)$ and "recede from" $(\dot{D}>0)$. Present day quantities are given the subscript zero. Alternative measures of distance are discussed in Appendix A

Figure 1illustrates the tethered galaxy problem. In an expanding universe distant galaxies recede with recession velocities given by Hubble's law, $v_{\text {rec }}=H D$, where $H$ is the time dependent Hubble constant $H=\dot{a} / a$. We adopt $H_{0}=70 \mathrm{~km} \mathrm{~s}^{-1} \mathrm{Mpc}^{-1}$. Suppose we separate a small test galaxy from the Hubble flow by tethering it to an observer's galaxy such that the proper distance between them remains constant. We neglect all practical considerations of such a tether because we can think of the tethered galaxy as one that has received a peculiar velocity boost toward the observer that exactly matches its recession velocity. We then remove the tether (or turn off the boosting rocket) to establish the initial condition of constant proper distance, $\dot{D}_{0}=0$. The idea of tethering is incidental, but for simplicity, we refer to this as the untethered or test galaxy. Note that this is an artificial setup; we have had to arrange for the galaxy to be moved out of the Hubble flow in order to apply this zero total velocity condition. Thus it is not a primordial condition, merely an initial condition that we have arranged for our experiment. Nevertheless, the discussion can be generalized to any object that has obtained a peculiar velocity and in Sec. IV we describe a similar situation that is found to occur naturally. We define the total velocity of the untethered galaxy as the time derivative of the proper distance, $v_{\text {tot }}=\dot{D}$,

$$
\begin{aligned}
\dot{D} & =\dot{a} \chi+a \dot{\chi} \\
v_{\text {tot }} & =v_{\text {rec }}+v_{\text {pec }} .
\end{aligned}
$$

The peculiar velocity $v_{\text {pec }}$ is the velocity with respect to the comoving frame out of which the test galaxy was boosted. It corresponds to our normal, local notion of velocity and must be less than the speed of light. In this section we consider only the nonrelativistic case, $v_{\text {pec }} \ll c$. The recession velocity $v_{\text {rec }}$ is the velocity of the Hubble flow at the proper distance $D$ and can be arbitrarily large 3.8 The motion of this test galaxy reveals the effect the expansion of the universe has on local dynamics. To enable us to isolate the effect of the expansion of the universe, we assume that the galaxies have negligible mass. By construction the tethered galaxy at an initial time $t_{0}$ has zero total velocity, $\dot{D}_{0}=0$, or

$$
\begin{aligned}
v_{\text {pec }, 0} & =-v_{\text {rec }, 0} \\
a_{0} \dot{\chi}_{0} & =-\dot{a}_{0} \chi_{0} .
\end{aligned}
$$

With this initial condition established, we untether the galaxy and let it coast freely. The question is then: Does the test galaxy approach, recede, or stay at the same distance?

The momentum $p$ with respect to the local comoving frame decays $\frac{18}{2}$ as $1 / a$. This scale factor dependent decrease in momentum is an important basis for many of the results that follow. For nonrelativistic velocities $p=m v_{\text {pec }}$ (for the relativistic solution see Appendix $[\mathbf{B}$ ), 


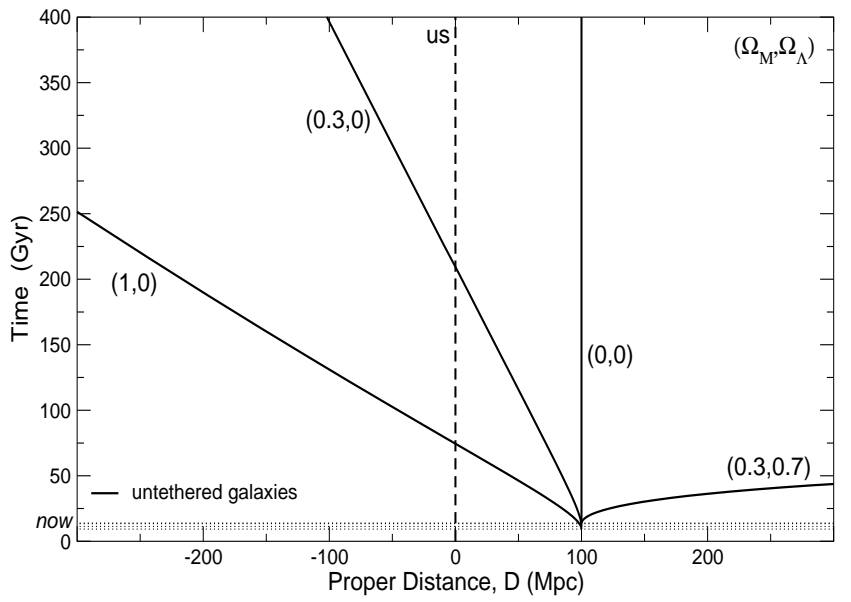

FIG. 2: Solutions to the tethered galaxy problem [Eq. 9]]. For four cosmological models we untether a galaxy at a distance of $D_{0}=100 \mathrm{Mpc}$ with an initial peculiar velocity equal to its recession velocity (total initial velocity is zero) and plot its path. In each case the peculiar velocity decays as $1 / a$. Its final position depends on the model. In the $\left(\Omega_{\mathrm{M}}, \Omega_{\Lambda}\right)=(0.3,0.7)$ accelerating universe, the untethered galaxy recedes from us as it joins the Hubble flow, while in the decelerating examples, $\left(\Omega_{\mathrm{M}}, \Omega_{\Lambda}\right)=(1,0)$ and $(0.3,0)$, the untethered galaxy approaches us, passes through our position and joins the Hubble flow in the opposite side of the sky. In the $\left(\Omega_{\mathrm{M}}, \Omega_{\Lambda}\right)=(0,0)$ model the galaxy experiences no acceleration and stays at a constant proper distance as it joins the Hubble flow [Eq. (15)]. In Sec. III and Fig. 5 we derive and illustrate the counter-intuitive result that such a galaxy will be blueshifted. We are the comoving galaxy represented by the thick dashed line labeled "us." There is a range of values labeled "now," because the current age of the universe is different in each model.

and, therefore,

$$
\begin{aligned}
v_{\mathrm{pec}} & =\frac{v_{\mathrm{pec}, 0}}{a} \\
a \dot{\chi} & =\frac{-\dot{a}_{0} \chi_{0}}{a} \\
\chi & =\chi_{0}\left[1-\dot{a}_{0} \int_{t_{0}}^{t} \frac{d t}{a^{2}}\right] \\
D & =a \chi_{0}\left[1-\dot{a}_{0} \int_{t_{0}}^{t} \frac{d t}{a^{2}}\right] .
\end{aligned}
$$

The integral in Eqs. (8) and (9) can be performed numerically by using $d t=d a / \dot{a}$ and $\dot{a}_{0}$, where both are obtained directly from the Friedmann equation,

$$
\dot{a}=\frac{d a}{d t}=H_{0}\left[1+\Omega_{\mathrm{M}}\left(\frac{1}{a}-1\right)+\Omega_{\Lambda}\left(a^{2}-1\right)\right]^{1 / 2} .
$$

The normalized matter density $\Omega_{\mathrm{M}}=8 \pi G \rho_{0} / 3 H_{0}^{2}$ and the cosmological constant $\Omega_{\Lambda}=\Lambda / 3 H_{0}^{2}$ are constants calculated at the present day. The scale factor $a(t)$ is derived by integrating the Friedmann equation 19

Equation (9) provides the general solution to the tethered galaxy problem. Figure 2 shows this solution for four different models. In the currently favored model, $\left(\Omega_{\mathrm{M}}, \Omega_{\Lambda}\right)=(0.3,0.7)$, the untethered galaxy recedes. In

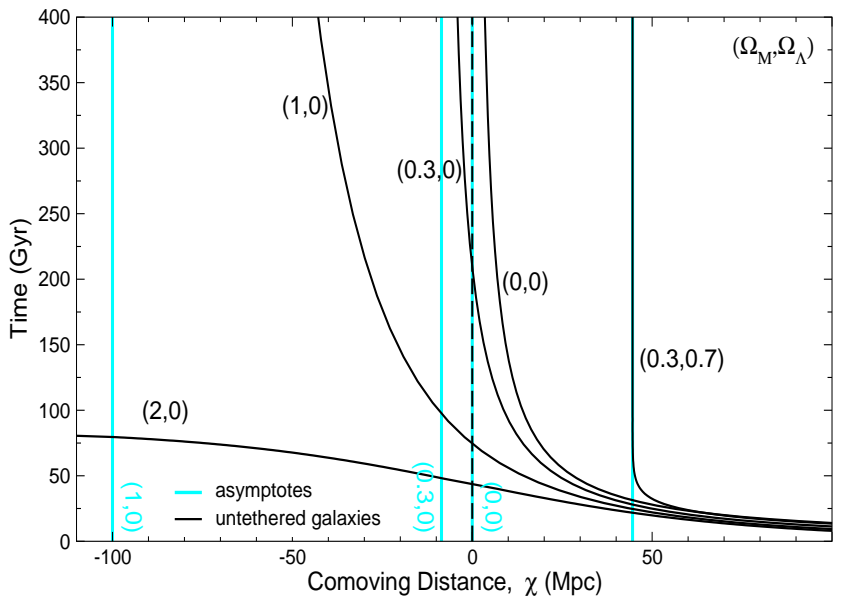

FIG. 3: Solutions to the tethered galaxy problem in comoving coordinates [Eq. 8] ] for five cosmological models. In all the models the comoving coordinate of the untethered galaxy decreases (our initial condition specified a negative peculiar velocity). In models that do not recollapse the untethered galaxy coasts and approaches an asymptote as it joins the Hubble flow. The rate of increase of the scale factor determines how quickly an object with a peculiar velocity joins the Hubble flow. In the accelerating universe $\left(\Omega_{\mathrm{M}}, \Omega_{\Lambda}\right)=(0.3,0.7)$, the perturbed galaxy joins the Hubble flow more quickly than in the decelerating universes $(1,0)$ and $(0.3,0)$, with the $(0,0)$ universe in between. The $\left(\Omega_{\mathrm{M}}, \Omega_{\Lambda}\right)=(2,0)$ model is the only model shown that recollapses. In the recollapsing phase of this model the galaxy's peculiar velocity increases as $a$ decreases and the galaxy does not join the Hubble flow [Eq. [B1]]. In the $(0,0)$ model the proper distance to the untethered galaxy is constant, and therefore its comoving distance $\chi=D / a$ tends toward zero (our position) as $a$ tends toward infinity. The different models have different starting points in time because the current age of the universe is different in each model.

the empty, $\left(\Omega_{\mathrm{M}}, \Omega_{\Lambda}\right)=(0,0)$ universe, it stays at the same distance while in the previously favored Einsteinde Sitter model, $\left(\Omega_{\mathrm{M}}, \Omega_{\Lambda}\right)=(1,0)$, and the $\left(\Omega_{\mathrm{M}}, \Omega_{\Lambda}\right)=$ $(0.3,0)$ model, it approaches. The different behavior in each model ultimately stems from the different compositions of the universes, because the composition dictates the acceleration. When the cosmological constant is large enough to cause the expansion of the universe to accelerate, the test galaxy will also accelerate away. When the attractive force of gravity dominates, decelerating the expansion, the test galaxy approaches. General solutions in comoving coordinates of the tethered galaxy problem are given by Eq. (8) and are plotted in Fig. 3 for the same four models shown in Fig. 2] as well as for a recollapsing model, $\left(\Omega_{\mathrm{M}}, \Omega_{\Lambda}\right)=(2,0)$.

\section{A. Expansion makes the untethered galaxy join the Hubble flow}

As shown in Fig. 3. the untethered galaxy asymptotically joins the Hubble flow in each cosmological model that expands forever. However, Fig. 2 shows that whether the untethered galaxy joins the Hubble flow by approaching or receding from us is a different, model 
dependent issue. The untethered galaxy asymptotically joins the Hubble flow for all cosmological models that expand forever because

$$
\dot{D}=v_{\mathrm{rec}}+v_{\mathrm{pec}}=v_{\mathrm{rec}}+v_{\mathrm{pec}, 0} / a .
$$

As $a \rightarrow \infty$ we have $\dot{D}=v_{\text {rec }}=H D$, which is pure Hubble flow. Note that the galaxy joins the Hubble flow solely due to the expansion of the universe ( $a$ increasing).

We further see that the expansion does not effect the dynamics because when we calculate the acceleration of the comoving galaxy, all terms in $\dot{a}$ cancel out:

$$
\begin{aligned}
\ddot{D} & =\dot{v}_{\mathrm{rec}}-\frac{v_{\mathrm{pec}, 0}}{a} \frac{\dot{a}}{a} \\
& =(\ddot{a} \chi+\dot{a} \dot{\chi})-\dot{a} \dot{\chi} \\
& =\ddot{a} \chi \\
& =-q H^{2} D,
\end{aligned}
$$

where the deceleration parameter $q(t)=-\ddot{a} a / \dot{a}^{2}$. Notice that the second term in Eq. (13) owes its existence to $\dot{\chi} \neq 0$ (which is only true if $v_{\text {pec }} \neq 0$ ) and here represents the galaxy moving to lower comoving coordinates. The resulting reduction in recession velocity is exactly canceled by the third term which is the decay of the peculiar velocity. Thus all terms in $\dot{a}$ cancel, and we conclude that the expansion, $\dot{a}>0$, does not cause acceleration, $\ddot{D}>0$. Thus, the expansion does not cause the untethered galaxy to recede (or to approach), but does result in the untethered galaxy joining the Hubble flow $\left(v_{\text {pec }} \rightarrow 0\right)$.

An alternative way to obtain Eq. (15) is to differentiate Hubble's Law, $\dot{D}=H D$. This method ignores $v_{\text {pec }}$ and therefore does not include the explicit cancellation of the two terms in Eq. (13) of the more general calculation. The fact that the results are the same emphasizes that the acceleration of the test galaxy is the same as that of comoving galaxies and there is no additional acceleration on our test galaxy pulling it into the Hubble flow.

\section{B. Acceleration of the expansion makes the untethered galaxy approach or recede}

Because the initial condition is $\dot{D}_{0}=0$, whether the galaxy approaches or recedes from us is determined by whether it is accelerated toward us $(\ddot{D}<0)$ or away from us $(\ddot{D}>0)$. Equation (15) shows that in an expanding universe, whether the galaxy approaches us or recedes from us does not depend on the velocity of the Hubble flow (because $H>0$ ) or the distance of the untethered galaxy (because $D>0$ ), but on the sign of $q$. When the universe accelerates $(q<0)$, the galaxy recedes from us. When the universe decelerates $(q>0)$, the galaxy approaches us. Finally, when $q=0$, the proper distance stays the same as the galaxy joins the Hubble flow. Thus the expansion does not "drag" the untethered galaxy away from us. Only the acceleration of the expansion can result in a change in distance between us and the untethered galaxy.

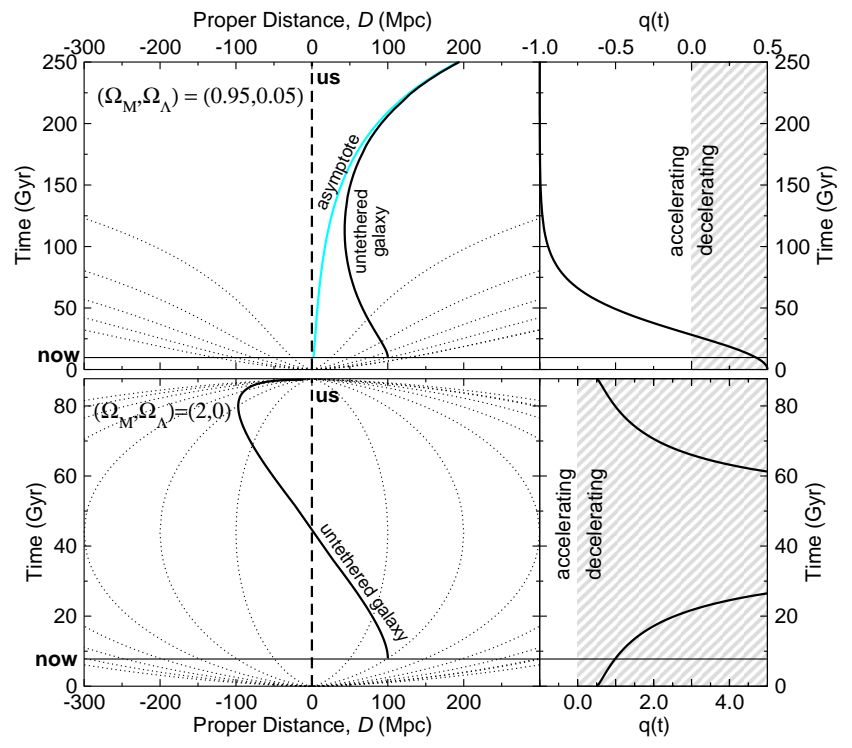

FIG. 4: Upper panels: The deceleration parameter $q(t)$ determines the acceleration of the untethered galaxy [Eq. [15] and can change sign. This particular model shows the effect of $q$ (right panel) on the position of the untethered galaxy (left panel). Initially $q>0$ and the proper distance to the untethered galaxy decreases [as in an $\left(\Omega_{\mathrm{M}}, \Omega_{\Lambda}\right)=(1,0)$ universe], but $q$ subsequently evolves and becomes negative, reflecting the fact that the cosmological constant begins to dominate the dynamics of the universe. With $q<0$, the acceleration $\ddot{D}$ changes sign. This makes the approaching galaxy slow down, stop, and eventually recede. The dotted lines are fixed comoving coordinates. Lower panels: The $\left(\Omega_{\mathrm{M}}, \Omega_{\Lambda}\right)=(2,0)$ universe expands and then recollapses ( $\dot{a}$ changes sign), and the peculiar velocity increases and approaches $c$ as $a \rightarrow 0$ [Eq. [B1].

Notice that in Eq. (15), $q=q(t)=q(a(t))$ is a function of the scale factor:

$q(a)=\left(\frac{\Omega_{\mathrm{M}}}{2 a}-\Omega_{\Lambda} a^{2}\right)\left[1+\Omega_{\mathrm{M}}\left(\frac{1}{a}-1\right)+\Omega_{\Lambda}\left(a^{2}-1\right)\right]^{-1}$,

which for $a\left(t_{0}\right)=1$ becomes the current deceleration parameter $q_{0}=\Omega_{\mathrm{M}} / 2-\Omega_{\Lambda}$. Thus, for example, the $\left(\Omega_{\mathrm{M}}, \Omega_{\Lambda}\right)=(0.66,0.33)$ model has $q_{0}=0$, but $q$ decreases with time, and therefore the untethered galaxy recedes. The upper panels of Fig. 4 show how a changing deceleration parameter affects the untethered galaxy. There is a time lag between the onset of acceleration $(q<0)$ and the galaxy beginning to recede $\left(v_{\text {tot }}>0\right)$ as is usual when accelerations and velocities are in different directions.

The example of an expanding universe in which an untethered galaxy approaches us exposes the common fallacy that "expanding space" is in some sense trying to drag all pairs of points apart. The fact that in the $\left(\Omega_{\mathrm{M}}, \Omega_{\Lambda}\right)=(1,0)$ universe the untethered galaxy, initially at rest, falls through our position and joins the Hubble flow on the other side of us does not argue against the idea of the expansion of space ${ }^{20}$ It does, however, highlight the common false assumption of a force or drag associated with the expansion of space. We have shown 
FIG. 5: The graphs show the combination of recession velocity and peculiar velocity that result in a redshift of zero, for four cosmological models. The purpose of these graphs is to display the counter-intuitive result that in an expanding universe a redshift of zero does not correspond to zero total velocity $(\dot{D}=0)$. Gray striped areas show the surprising situations where receding galaxies appear blueshifted or approaching galaxies appear redshifted. Other models [for

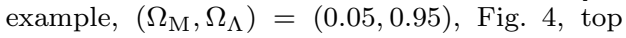
panel] can have both approaching redshifted and receding blueshifted regions simultaneously. Recession velocities are calculated at the time of emission; the results are qualitatively the same when recession velocities are calculated at the time of observation. Thus galaxies that were receding at emission and are still receding, can be blueshifted. Note that in each panel for low velocities (nearby galaxies), the $z_{\text {tot }}=0$ line asymptotes to the $v_{\text {tot }}=0$ line. See Sec. IV for a discussion of the active galactic nuclei jet

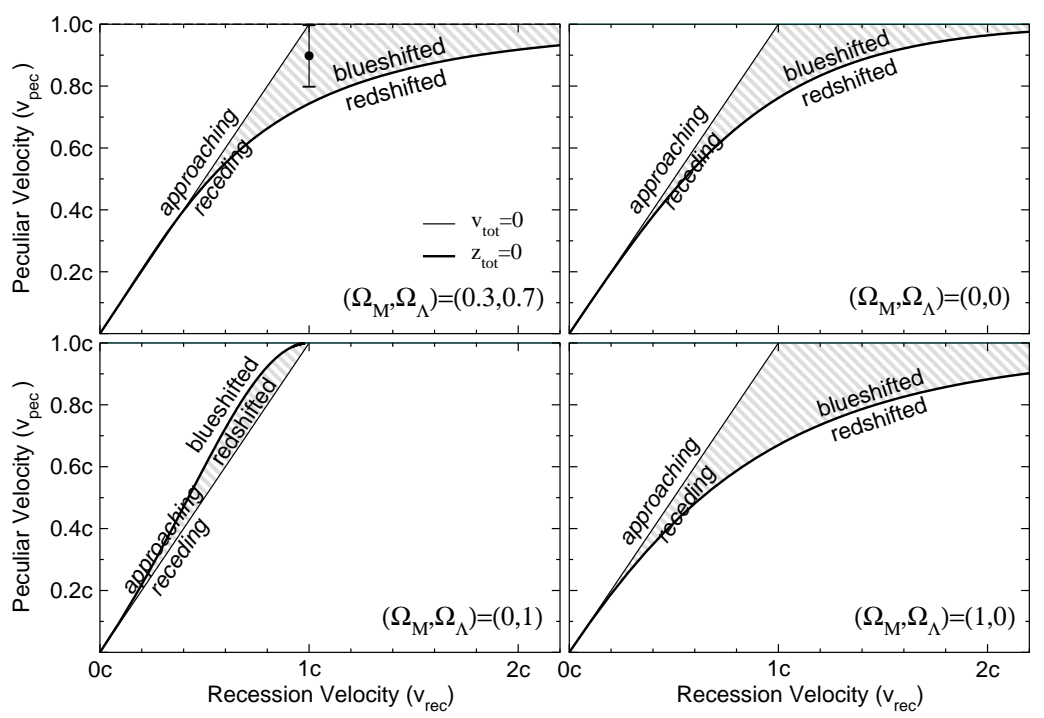
data point in the upper left panel.

that an object with a peculiar velocity does rejoin the Hubble flow in eternally expanding universes, but does not feel any force causing it to rejoin the Hubble flow. This qualitative result extends to all objects with a peculiar velocity.

\section{A TETHERED GALAXY HAS A NONZERO REDSHIFT}

In the context of special relativity (Minkowski space), objects at rest with respect to an observer have zero redshift. However, in an expanding universe special relativistic concepts do not generally apply. "At rest" is defined to be "at constant proper distance" $\left(v_{\text {tot }}=\dot{D}=0\right)$, so our untethered galaxy with $\dot{D}_{0}=0$ satisfies the condition for being at rest. Will it therefore have zero redshift? That is, are $z_{\text {tot }}=0$ and $v_{\text {tot }}=0$ equivalent? Although radial recession and peculiar velocities add vectorially, their corresponding redshift components combine ${ }^{23}$ as $\left(1+z_{\text {tot }}\right)=\left(1+z_{\text {rec }}\right)\left(1+z_{\text {pec }}\right)$. The condition that $z_{\text {tot }}=0$ gives

$$
\left(1+z_{\mathrm{pec}}\right)=\frac{1}{\left(1+z_{\mathrm{rec}}\right)} .
$$

The special relativistic relation between peculiar velocity and Doppler redshift is

$$
v_{\mathrm{pec}}\left(z_{\mathrm{pec}}\right)=c\left[\frac{\left(1+z_{\mathrm{pec}}\right)^{2}-1}{\left(1+z_{\mathrm{pec}}\right)^{2}+1}\right],
$$

while the general relativistic relation between recession velocity (at emission 24 ) and cosmological redshift is 21

$$
v_{\mathrm{rec}}\left(z_{\mathrm{rec}}\right)=c \frac{H\left(z_{\mathrm{rec}}\right)}{1+z_{\mathrm{rec}}} \int_{0}^{z_{\mathrm{rec}}} \frac{d z}{H(z)},
$$

where $H\left(z_{\mathrm{rec}}\right)=H\left(t_{\mathrm{em}}\right)$ is Hubble's constant at the time of emission. Hubble's constant as a function of cosmological redshift is obtained by rearranging Friedmann's equation [Eq. (10)],

$$
H(z)=H_{0}(1+z)\left[1+\Omega_{\mathrm{M}} z+\Omega_{\Lambda}\left(\frac{1}{(1+z)^{2}}-1\right)\right]^{1 / 2} .
$$

In Fig. [5 we plot the $v_{\text {tot }}=0$ and the $z_{\text {tot }}$ lines to show they are not coincident. To obtain the $z_{\text {tot }}=0$ curve, we do the following: For a given $v_{\text {rec }}$ we use Eq. (19) to calculate $z_{\text {rec }}$ (for a particular cosmological model). Equation (17) then gives us a corresponding $z_{\text {pec }}$ and we can solve for $v_{\text {pec }}$ using Eq. (18). The result is the combination of peculiar velocity and recession velocity required to give a total redshift of zero. The fact that the $z_{\text {tot }}=0$ curves are different from the $v_{\text {tot }}=0$ line in all models shows that $z_{\text {tot }}=0$ is not equivalent to $v_{\text {tot }}=0$. Recession velocities due to expansion have a different relation to the observed redshift [Eq. (19)] than do peculiar velocities [Eq. [18] ] $]^{3}$

That the $z_{\text {tot }}=0$ line is not the same as the $v_{\text {tot }}=0$ line even in the $q=0,\left(\Omega_{\mathrm{M}}, \Omega_{\Lambda}\right)=(0,0)$ model (upper right Fig. (5) is particularly surprising because we might expect an empty expanding FRW universe to be welldescribed by special relativity in flat Minkowski spacetime. Zero velocity approximately corresponds to zero redshift for $v_{\text {rec }} \lesssim 0.3 c$ or $z_{\text {rec }} \lesssim 0.3$ [not just for the $(0,0)$ model but for all models], but for larger redshifts is not the case because of the different way time is defined in the FRW and Minkowski metrics. A coordinate change can be made to make the FRW model look like Minkowski spacetime, but the homogeneity of constant time surfaces is lost $\frac{9}{\underline{9}}$ As a consequence, in the $\left(\Omega_{\mathrm{M}}, \Omega_{\Lambda}\right)=(0,0)$ model, a galaxy at a constant distance $(\dot{D}=0)$ will be blueshifted. An analytical derivation of the solution for the empty universe is given in Appendix C 
The fact that approaching galaxies can be redshifted and receding galaxies can be blueshifted is an interesting illustration of the fact that cosmological redshifts are not Doppler shifts. The expectation that when $v_{\text {tot }}=0$, $z_{\text {tot }}=0$, comes from special relativity and does not apply to galaxies in the general relativistic description of an expanding universe, even an empty one.

\section{OBSERVATIONAL CONSEQUENCES}

The result for the tethered galaxy can be applied to the related case of active galactic nuclei outflows. Some compact extragalactic radio sources at high redshift are seen to have bipolar outflows of relativistic jets of plasma. Jets directed toward us (and in particular the occasional knots in it) are analogs of a tethered (or boosted) galaxy. These knots have peculiar velocities in our direction, but their recession velocities are in the opposite direction and can be larger. Thus the proper distance between us and the knot can be increasing. They are receding from us (in the sense that $\dot{D}>0$ ), yet, as we have shown here, the radiation from the knot can be blueshifted. In Fig. 6 the zero-total-velocity condition is plotted in terms of the observable redshifts of a central-source and jet system.

We can predict which radio sources have receding blueshifted jets. The radio source $1146+531$, for example, has a redshift $z_{\text {rec }}=1.629 \pm 0.0052^{22}$ In an $\left(\Omega_{\mathrm{M}}, \Omega_{\Lambda}\right)=(0.3,0.7)$ universe, its recession velocity at the time of emission was $v_{\text {rec }} \approx c$. Therefore the relativistic jet $\left(v_{\text {pec }}<c\right)$ it emits in our direction was (and is) receding from us and yet, if the parsec scale jet has a peculiar velocity within the typical estimated range $0.8 \lesssim v_{\text {pec }} / c \lesssim 0.99$, it will be blueshifted. This example is the point plotted in the upper left panel of Fig. 5 .

\section{SUMMARY}

We have pointed out and interpreted some counterintuitive results of the general relativistic description of our Universe. We have shown that the unaccelerated expansion of the universe has no effect on whether an untethered galaxy approaches or recedes from us. In a decelerating universe the galaxy approaches us, while in an accelerating universe the galaxy recedes from us. The expansion, however, is responsible for the galaxy joining the Hubble flow, and we have shown that this happens whether the untethered galaxy approaches or recedes from us.

The expansion of the universe is a natural feature of general relativity that also allows us to unambiguously convert observed redshifts into proper distances and recession velocities and to unambiguously define approach and recede. We have used this foundation to predict the existence of receding blueshifted and approaching redshifted objects in the universe. To our knowledge this is the first explicit derivation of this counter-intuitive behavior.

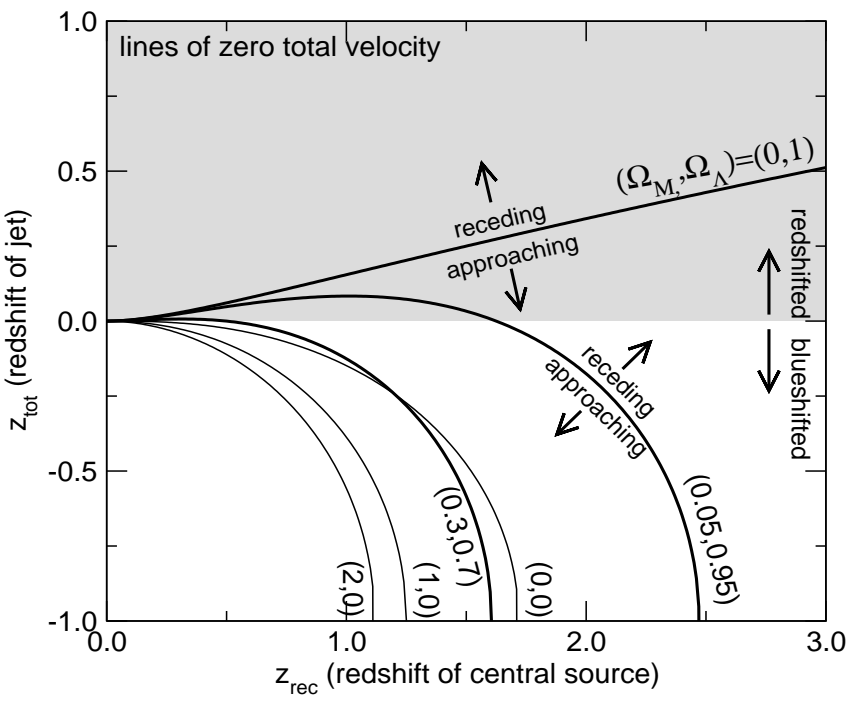

FIG. 6: This graph expresses the same information as Fig. 1 but in terms of observables. An active galactic nuclei with the central source of cosmological redshift, $z_{\mathrm{rec}}$, is assumed to be comoving. The observed redshift of a knot in a jet, $z_{\text {tot }}$, is the total redshift resulting from the peculiar velocity of the jet and from the cosmological redshift. The $z_{\text {tot }}=0$ boundary separates the redshifted region (upper) from the blueshifted region (lower). The curves correspond to a total velocity of zero $(\dot{D}=0)$ for different models, $\left(\Omega_{\mathrm{M}}, \Omega_{\Lambda}\right)$, as labeled. The regions representing receding objects and approaching objects are indicated for the $\left(\Omega_{\mathrm{M}}, \Omega_{\Lambda}\right)=(0.05,0.95)$ and $\left(\Omega_{\mathrm{M}}, \Omega_{\Lambda}\right)=(0,1)$ models as examples (recession or approach at emission is plotted). In contrast, for expectations based on special relativity, receding objects are not necessarily redshifted, nor are blueshifted objects necessarily approaching us.

Concepts such as "recede" or "approach" and quantities such as $\dot{D}$ are of limited use in observational cosmology because all our observations come to us via the backward pointing null cone. This limitation will remain the case until a very patient observer organizes a synchronized set of comoving observers to measure proper distance 17 However, the issue we are addressing - the relationship between observed redshifts and expansion - is a conceptual one and is closely related to the important conceptual distinction between the theoretical and empirical Hubble laws ${ }^{3} \underline{3}$

\section{Postscript:}

Post publication it was brought to our attention that $\mathrm{T}$. Kiang has published a similar analysis of the redshifts of relativistic jets. His excellent paper can be found in Chinese Astronomy and Astrophysics 25, 141-146 (2001).

\section{Acknowledgments}

TMD is supported by an Australian Postgraduate Award. CHL acknowledges an Australian Research Council Fellowship. JKW acknowledges useful discussions with Ken Lanzetta. We wish to thank Jochen Liske, John Peacock, Phillip Helbig, Edward Harrison, Joe Wolfe, and Frank Tipler for useful discussions and two anonymous referees for constructive comments. 


\section{APPENDIX A: LUMINOSITY DISTANCE, ANGULAR-DIAMETER DISTANCE}

The FRW metric including the angular terms is

$$
d s^{2}=-c^{2} d t^{2}+a^{2}(t)\left[d \chi^{2}+S_{k}^{2}(\chi)\left(d \theta^{2}+\chi^{2} d \phi^{2}\right)\right],
$$

where $S_{k}(\chi)=\sinh \chi, \chi, \sin \chi$ for $k=-1,0,1$, respectively, and $\theta$ and $\phi$ are the angular measures in spherical coordinates. We use the proper distance $D=a \chi$, which is the distance measured along a spatial geodesic, the path light follows through space. Other distance measures in common use are angular diameter distance $D_{A}=(1+z)^{-1} a(t) S_{k}(\chi)$ and luminosity distance $D_{L}=$ $(1+z) a(t) S_{k}(\chi)$. Both include the $S_{k}$ term, which means they both involve the distance perpendicular to the line of sight. $\left[S_{k}(\chi)\right.$ appears only in the metric when multiplied by an angular term.] They can be used to parametrize distance, but have no direct relation to recession velocity and cannot be used to explain the observed redshift. The distance in Hubble's law, $v_{\text {rec }}=H D$, is proper distance. If one prefers to use $D_{A}$ or $D_{L}$ as measures of distance and $\dot{D}_{L}$ and $\dot{D}_{A}$ to define "approach" and "recede," it can also be shown, using the relationships between $\dot{D}$, $\dot{D}_{A}$, and $\dot{D}_{L}$, in a fashion similar to what we have done for proper distance, that $z_{\text {tot }}=0$ is not equivalent to either $\dot{D}_{L}=0$ or $\dot{D}_{A}=0$.

\section{APPENDIX B: RELATIVISTIC SOLUTION FOR PECULIAR VELOCITY}

When a universe collapses, the scale factor $a$ decreases. Thus $v_{\text {pec }} \propto 1 / a$ [see Eq. [6)] means that the peculiar velocity increases with time. Therefore, in collapsing universes, untethered galaxies do not "join the Hubble flow." This behavior is shown for the $\left(\Omega_{\mathrm{M}}, \Omega_{\Lambda}\right)=(2,0)$ model in Fig. 3 Collapsing universes require the relativistic formula for the change of the peculiar velocity to avoid the infinite peculiar velocities that result from $v_{\text {pec }} \propto 1 / a$ as $a \rightarrow 0$. To produce all the figures in this paper, except the lower panels in Fig. [ we have used $p=m v_{\text {pec }}$. However, as the peculiar velocities become relativistic in a collapsing universe, we need to use the special relativistic formula for momentum $p=\gamma m v_{\text {pec }}$, where $\gamma=\left(1-v_{\text {pec }}^{2} / c^{2}\right)^{-1 / 2}$. Because momentum decays as $1 / a\left(p=p_{\mathrm{o}} a_{0} / a\right)$, we obtain,

$$
v_{\mathrm{pec}}=\frac{\gamma_{0} v_{\mathrm{pec}, 0}}{\sqrt{a^{2}+\gamma_{0}^{2} v_{\mathrm{pec}_{0}}^{2} / c^{2}}} .
$$

Therefore, as $a \rightarrow 0, v_{\text {pec }} \rightarrow c$. Equation (B1) was used

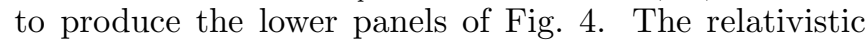
formula for momentum should also be used in eternally expanding universes if relativistic velocities are set as the initial condition in Eq. (4). Using Eq. (B1) in Eq. (11) results in a residual dependence on $\dot{a}$ in Eq. (14). The residual is negligible for $v \ll c$, and becomes negligible for $v \sim c$ as $a \rightarrow \infty$. Note that Eq. (18) is relativistic and therefore the results of Section III hold for $v_{\text {pec }} \sim c$.

Collapsing universes also provide the possibility of approaching-redshifted objects, but without involving peculiar velocities. In the collapsing phase all galaxies are approaching us. However, if the galaxy is distant enough, it may have been receding for the majority of the time its light took to propagate to us. In this case the galaxy appears redshifted even though it may be approaching at the time of observation. This example differs from the active galactic nuclei jet example because the active galactic nuclei jet may appear blueshifted even though the jet never approaches us.

\section{APPENDIX C: ANALYTIC SOLUTION FOR THE EMPTY UNIVERSE}

In the empty $\left(\Omega_{\mathrm{M}}, \Omega_{\Lambda}\right)=(0,0)$ universe, an analytical solution can be found for the combination of recession and peculiar velocity that would give a redshift of zero. For an empty expanding universe, $H(z)=H_{0}(1+z)$, and the time derivative of the scale factor at emission is $\dot{a}_{\mathrm{em}}=H_{0}$. Therefore, Eq. (19) becomes

$$
\begin{aligned}
v_{\text {rec }} & =c H_{0} \int_{0}^{z_{\text {rec }}} \frac{d z}{H_{0}(1+z)} \\
& =c \ln \left(1+z_{\text {rec }}\right) \\
e^{v_{\text {rec }} / c} & =1+z_{\text {rec }} .
\end{aligned}
$$

If we substitute Eq. (C3) into Eq. (17) followed by Eq. (18), we find

$$
v_{\mathrm{pec}}=c\left[\frac{e^{-2 v_{\mathrm{rec}} / c}-1}{e^{-2 v_{\mathrm{rec}} / c}+1}\right] .
$$

Equation (C4) shows that only in the limit of small $v_{\text {rec }}$ does $v_{\text {pec }}=-v_{\text {rec }}$ for $z_{\text {tot }}=0$. Equation (C4) generates the thick black $z=0$ line in Fig. 5 . upper right panel.

\section{APPENDIX D: SUGGESTED PROBLEMS}

The host galaxy of active galactic nuclei $1146+531$ has a redshift $z_{\text {rec }}=1.63$. Assume for simplicity that we live in a universe with $\left(\Omega_{\mathrm{M}}, \Omega_{\Lambda}\right)=(1,0)$.

(a) What was the galaxy's recession velocity at the time it emitted the light we now see? [Refer to Eqs. (19) and (20).]

(b) If the jet it emits had a peculiar velocity in our direction of $v_{\text {pec }}=0.80 c$, what was the jet's total velocity at the time of emission? [Refer to Eq. (3).] Is it moving away from or toward us?

(c) What is the jet's total redshift, $z_{\text {tot }}$ ? [See Eq. (18) and the text preceding Eq. [17).] Is it redshifted or blueshifted?

(d) What is the galaxy's recession velocity at the time of observation? ${ }^{24}$ Compared to your answer in Part (a) is this behavior what you would expect for a decelerating universe? 
* Electronic address: tamarad@phys.unsw.edu.au

$\dagger$ Electronic address: charley@bat.phys.unsw.edu.au

¥ Electronic address: jkw@bat.phys.unsw.edu.au

1 H. P. Robertson, "Kinematics and world structure," Astrophys. J. 82, 284-301 (1935).

2 A. G. Walker, "On Milne's theory of world-structure," Proc. Lond. Math. Soc. 2 (42), 90-127 (1936).

3 E. R. Harrison, "The redshift-distance and velocitydistance laws," Astrophys. J. 403, 28-31 (1993).

4 E. A. Milne, "A Newtonian expanding universe," Q. J. Math. Oxford 5, 64-72 (1934), reprinted in Gen. Relativ. Gravit. 32 (9), 1939-1948 (2000); W. H. McCrea and E. A. Milne, "Newtonian universes and the curvature of space," Q. J. Math. Oxford 5, 73-80 (1934), reprinted in Gen. Relativ. Gravit. 32 (9), 1949-1958 (2000).

5 D. Layzer, "On the significance of Newtonian cosmology," Astron. J. 59 (1219), 268-269 (1954).

${ }^{6}$ F. J. Tipler, "Newtonian cosmology revisited," Mon. Not. R. Astron. Soc. 282, 206-210 (1996); F. J. Tipler, "Rigorous Newtonian cosmology," Am. J. Phys. 64 (10), 13111315 (1996); see also G. Endean, "Redshift and the Hubble constant in conformally flat spacetime," Astrophys. J. 434, 397-401 (1994) refuted by L. Querella, "Kinematic cosmology in conformally flat spacetime," ibid 508, 129131 (1998).

7 G. C. McVittie, "Distance and large redshifts," Q. J. R. Astron. Soc. 15, 246-263 (1974); S. Weinberg, The First Three Minutes (Bantum Books, London, 1977), pp. 12, 25, 27; B. F. Schutz, A First Course in General Relativity (Cambridge U. P., Cambridge, 1985), p. 320; P. J. E. Peebles, D. N. Schramm, E. L. Turner, and R. G. Kron, "The case for the relativisitc hot big bang cosmology," Nature (London) 352, 769-776 (1991).

8 See for example, H. S. Murdoch, "Recession velocities greater than light," Q. J. R. Astron. Soc. 18, 242-247 (1977); W. M. Stuckey, "Can galaxies exist within our particle horizon with Hubble recessional velocities greater than $c$ ?," Am. J. Phys 60 (2), 142-146 (1992); T. Kiang, "Horizon and the question whether galaxies that recede faster than light are observable," Chin. Astron. Astrophys. 21 (1), 1-18 (1997).

9 D. N. Page, "No superluminal expansion of the universe," gr-qc/9303008

10 J. A. Peacock, Cosmological Physics (Cambridge U. P., Cambridge, 1999), pp. 6, 87-88; the form of FriedmannRobertson-Walker metric is on p. 70.

11 F. Munley, "Question \#15: What space scales participate in cosmic expansion?," Am. J. Phys. 63 (4), 297 (1995); and subsequent answers from S. Blau, ibid 63 (9), 779780 (1995); C. Callender, ibid 63 (9), 780 (1995); J. L. Anderson, ibid 64 (5), 527-528 (1996). Also A. Harvey, "Space is expanding," ibid 56 (6), 487-488 (1988).

12 X. Shi and M. S. Turner, "Expectations for the difference between local and global measurements of the Hubble constant," Astrophys. J. 493, 519-522 (1998); F. J. Tipler, "How far out must we go to get into the Hubble Flow?," ibid 511, 546-549 (1999); T. Chiueh and X. $\mathrm{He}$, "Future island universes in a background universe accelerated by cosmological constant and by quintessence," astro-ph/0107453 (2001); Y. V. Dumin, "On a probable manifestation of Hubble expansion at the local scales, as inferred from LLR data," astro-ph/0203151 (2002).

13 O. Lahav, P. B. Lilje, J. R. Primack, and M. J. Rees, "Dynamical effects of the cosmological constant," Mon. Not. R. Astron. Soc. 251, 128-136 (1991); J. L. Anderson, "Multiparticle dynamics in an expanding universe," Phys. Rev. Lett. 75 (20), 3602-3604 (1995); F. K. Cooperstock, V. Faraoni, and D. N. Vollick, "The influence of the cosmological expansion on local systems," Astrophys. J. 503, 61-66 (1998); A. J. S. Hamilton, "Formulae for growth factors in expanding universes containing matter and a cosmological constant," Mon. Not. R. Astron. Soc. 322, 419-425 (2001); G. A. Baker, Jr., "Effect on the structure of the universe of an accelerating expansion," Gen. Relativ. Gravit. 34, 767-791 (2002); astro-ph/0112320

14 A. N. Silverman, "Resolution of a cosmological paradox using concepts from general relativity theory," Am. J. Phys. 54 (12), 1092-1096 (1986); W. M. Stuckey, "Can galaxies exist within our particle horizon with Hubble recessional velocities greater than $c$ ?," ibid 60 (2), 142-146 (1992); W. M. Stuckey, "Kinematics between comoving photon exchangers in a closed, matter-dominated universe," ibid 60 (6), 554-560 (1992); G. F. R. Ellis and T. Rothman, "Lost horizons," ibid 61 (10), 883-893 (1993); F. J. Tipler, "Rigorous Newtonian cosmology," ibid 64 (10), 1311-1315 (1996).

15 E. R. Harrison, "Mining energy in an expanding universe," Astrophys. J. 446, 63-66 (1995).

16 J. A. Peacock, in Como 2000 Proceedings of the School on Relativistic Gravitation (Springer, New York, 2001), Sec.1.4.7.

17 S. Weinberg, Gravitation and Cosmology (John Wiley \& Sons, New York, 1972), p. 415; W. Rindler, Essential Relativity, Special, General and Cosmological (Springer-Verlag, New York, 1977), p. 218.

18 C. W. Misner, K. S. Thorne. and J. A. Wheeler, Gravitation (Freeman, San Francisco, 1970), Ex. 29.3; S. Weinberg, Gravitation and Cosmology (Wiley, New York, 1972), p. 415; P. J. E. Peebles, Principles of Physical Cosmology, (Princeton U. P., Princeton, 1993), pp. 93-99; T. Padmanabhan, Cosmology and Astrophysics Through Problems (Cambridge U. P., Cambridge, 1996), Sec. 6.2c.

19 J. E. Felten and R. Isaacman, "Scale factors $R(t)$ and critical values of the cosmological constant $\Lambda$ in Friedmann universes," Rev. Mod. Phys. 58, 689-698 (1986).

20 See Peacock 1999 (Ref. 10) and Peacock 2001 (Ref. 16) for a different interpretation.

21 E. R. Harrison, "The redshift-distance and velocitydistance laws," Astrophys. J. 403, 28-31 (1993), Eq. 13.

22 R. C. Vermeulen and G. B. Taylor, "Redshifts for superluminal candidates," Astron. J. 109(5), 1983-1987 (1995).

23 Light is emitted by the tethered galaxy. Let $\lambda_{\text {observed }}$ be the wavelength we observe, $\lambda_{\text {emitted }}$ be the wavelength measured in the comoving frame of the emitter (the frame with respect to which it has a peculiar velocity $\left.v_{\text {pec }}\right)$ and $\lambda_{\text {rest }}$ be the wavelength of light in the rest frame of the emitter. Then $1+z_{\text {tot }}=\frac{\lambda_{\text {observed }}}{\lambda_{\text {rest }}}=\frac{\lambda_{\text {observed }}}{\lambda_{\text {emitted }}} \frac{\lambda_{\text {emitted }}}{\lambda_{\text {rest }}}=$ $\left(1+z_{\text {rec }}\right)\left(1+z_{\mathrm{pec}}\right)$.

24 To calculate the current recession velocity (as opposed to the recession velocity at the time of emission) replace $z_{\text {rec }}$ with $z=0$ in Eq. (19) (except in the upper limit of the integral). 